\title{
Necrotizing Fasciitis is a Rare Complication after Bone Marrow Transplantation: A Case Report
}

\author{
Mehrdad Payandeh ${ }^{1}$, Mohammad Hossein Zamanian², Cyrus Rezaei ${ }^{3}$, Sakineh Ghorbani ${ }^{4}$, \\ Mehrnoush Aeinfar ${ }^{5}$, Masoud Sadeghi,"* \\ ${ }^{1}$ Department of Hematology and Medical Oncology, Kermanshah University of Medical Sciences, Kermanshah, Iran \\ ${ }^{2}$ Department of Infectious Diseases and Tropical Medicine, Kermanshah University of Medical Sciences, Kermanshah, Iran \\ ${ }^{3}$ Department of Orthopedics, Kermanshah University of Medical Sciences, Kermanshah, Iran \\ ${ }^{4}$ Department of Nursing, Kermanshah University of Medical Sciences, Kermanshah, Iran \\ ${ }^{5}$ Medical Biology Research Center, Kermanshah University of Medical Sciences, Kermanshah, Iran \\ *Corresponding author: Sadeghi_mbrc@yahoo.com
}

\begin{abstract}
Necrotizing fasciitis is an uncommon disease and a soft tissue infection that spreads along fascial planes with associated inflammation and necrosis. Herein, this study reported necrotizing fasciitis as a rare complication after bone marrow transplantation (BMT) in a Iranian patient and the efficacy of early treatment in improvement it. A 28-year-old lady had known case of first relapse of Hodgkin's lymphoma that admitted for auto-BMT in 2016. Five years ago due to abdominal-pelvic massive lymphadenopathy, she was treated with six courses of ABVD regimen combined with involved filed irradiation. Due to Hodgkin's relapse, the patient was treated with six courses of ICE regimen and after complete remission was selected for auto-BMT. Necrotizing fasciitis was reported by MRI and the patients was treated with combination of a number of antibiotics after fasciectomy. In conclusion, necrotizing fasciitis is a rare complication of BMT, especially autologous type that drug selection and rapid surgical fasciectomy are important decisions for prevention of mortality and morbidity of this complication.
\end{abstract}

Keywords: necrotizing fasciitis, Bone marrow transplantation, Hodgkin's disease

Cite This Article: Mehrdad Payandeh, Mohammad Hossein Zamanian, Cyrus Rezaei, Sakineh Ghorbani, Mehrnoush Aeinfar, and Masoud Sadeghi, "Necrotizing Fasciitis is a Rare Complication after Bone Marrow Transplantation: A Case Report." American Journal of Cancer Prevention, vol. 5, no. 2 (2017): 28-30. doi: 10.12691/ajcp-5-2-3.

\section{Introduction}

Necrotizing fasciitis is an uncommon disease and is considered a rare and potentially fatal condition [1,2] that results in gross morbidity and mortality if not diagnosed and treated in its early stages [3,4]. Necrotizing fasciitis is a soft tissue infection that spreads along fascial planes with associated inflammation and necrosis [1]. Necrotizing fasciitis has been historically reported from almost all parts of the world and is now understood to be caused by either a single organism or more frequently by a variety of microbes-both aerobic and anaerobic [5]. The incidence of necrotizing fasciitis has been varyingly reported worldwide, 0.4 to 7.45 cases per 100,000 population and also the mortality rates reported from 15 to $34 \%$ [6]. The aim of this study was to report necrotizing fasciitis as a rare complication after bone marrow transplantation (BMT) in a Iranian patient (Western Iran) and the efficacy of early treatment in improvement it.

\section{Case Report}

A 28-year-old female had known case of first relapse (second remission) of Hodgkin's lymphoma that admitted for auto-BMT in 2016. Her disease was presented at first time with abdominal-pelvic massive lymphadenopathy from five years ago and she was treated with 6 courses of ABVD regimen (doxorubicin, bleomycin, vinblastine and dacarbazine) combined with involved filed irradiation. During this five-year follow-up was good, but she in last visit was presented with abdominal pain and right leg pitting edema (Figure 1). After further evaluation in biopsy with a diagnosis of Hodgkin's relapse was treated with six courses of ICE (Ifosfamide, carboplatin and etoposide) regimen and after complete remission was selected for auto-BMT. Two days after cell separation and conditioning with BEAM (BCNU (carmustine), etoposide, cytrabine and melphalan) regimen, progressive left leg presented with diffuse redness and tensive tenderness. With a diagnosis of cellulites during profound neutropenia, she was treated with a combination of meropenem, vancomycin and clindamycin, but despite of these potent intensive, drug therapy was not seen a very effective response. On left leg magnetic resonance imaging (MRI), necrotizing fasciitis was reported. She referred for fasciectomy, and previous drug regimens changed to combination of lenizolide, levofluxacin and meropenem. Due to profound prolonged neutropenia, more than 14 days prophylaxis with voriconazole was recommended 
during this time. At the end, she was discharged 25 days after cell transplantation with good organ function and elimination of progressive fasciitis (Figure 2).

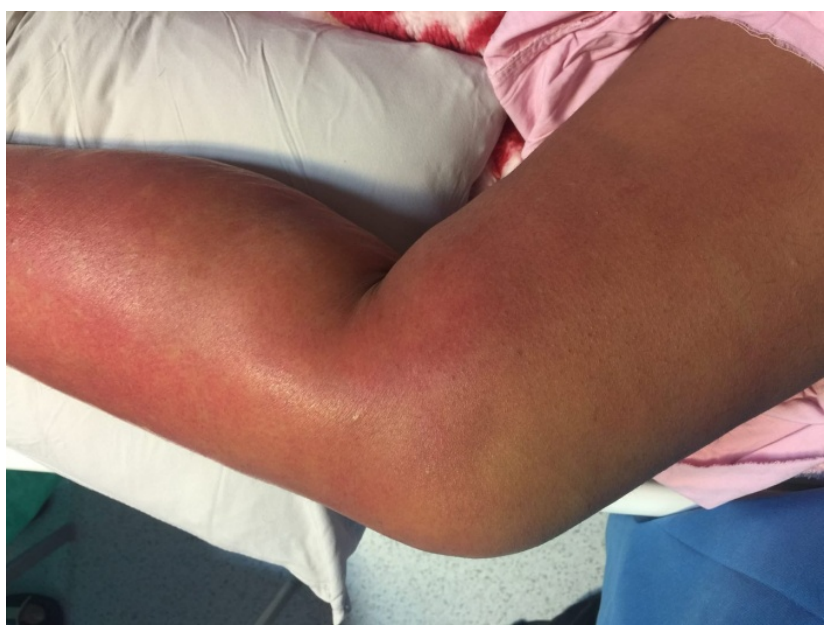

Figure 1. Before treatment

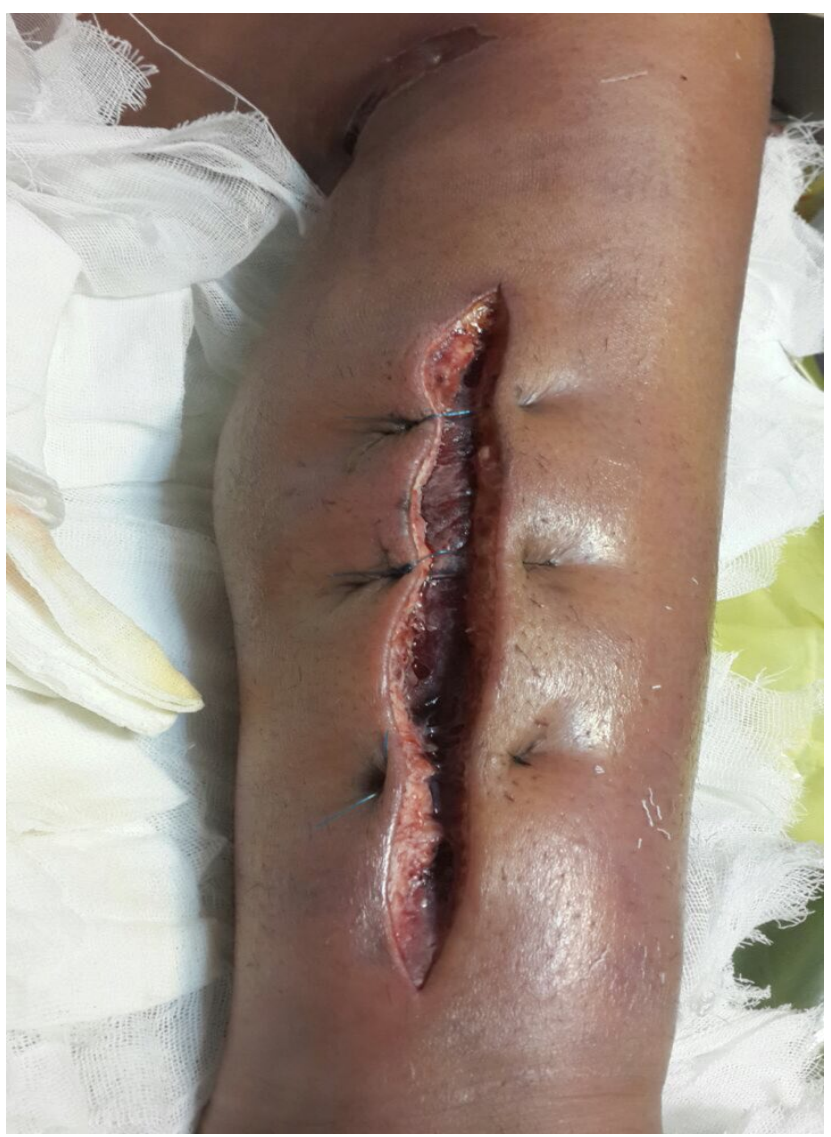

Figure 2. After treatment

\section{Discussion}

Necrotizing fasciitis is a rare and progressive bacterial infection that affects all age groups, although middle-aged and elderly patients (over 50 years of age) are more likely to be infected [7]. Necrotizing fasciitis is uncommon and difficult to diagnose and it causes progressive morbidity until the infectious process is diagnosed and treated medically and surgically [8]. The early clinical diagnosis of an area of necrotizing fasciitis is difficult and frequently unreliable [9], but early diagnosis of this disorder and prompt initiation of appropriate therapy can prevent progression of this acute necrotizing infection $[10,11]$ and reduce the severity, morbidity and mortality [12]. The mortality rates are still very high in necrotizing fasciitis due to the severe sepsis that necessitates other interventions to overcome sepsis-related mortality [8]. Even with optimal treatment, the overall mortality rate is $15 \%$ to $20 \%$ [1]. The first treatment is mainly surgical with relevant early radical debridement of devitalized tissue [13]. As a general rule, necrotizing fasciitis without surgical intervention has a mortality rate of $100 \%$ [14]. Preadmission treatment with antibiotics modified the initial clinical picture and often masked the severity of the underlying infection [11]. Therefore, the main treatment of necrotizing fasciitis is a combination of surgical debridement, appropriate antibiotics and optimal oxygenation of the infected tissues $[8,11,15,16]$ that also our case was treated with combination of fasciectomy and several antibiotics, whatever the treatment was heavy for the patients, but the result was successful and eliminated of progressive fasciitis.

\section{Conclusion}

Necrotizing fasciitis is a rare complication of BMT, especially autologous type that drug selection and rapid surgical fasciectomy are important decisions for prevention of mortality and morbidity of this complication.

\section{References}

[1] Anaya DA, McMahon K, Nathens AB, Sullivan SR, Foy H, Bulger E. Predictors of mortality and limb loss in necrotizing soft tissue infections. Arch Surg. 2005; 140(2): 151-7.

[2] Navinan MR, Yudhishdran J, Kandeepan T, Kulatunga A. Necrotizing fasciitis--a diagnostic dilemma: two case reports. J Med Case Rep. 2014; 8: 229.

[3] Puvanendran R, Huey JC, Pasupathy S. Necrotizing fasciitis. Can Fam Physician. 2009; 55(10):981-7.

[4] Cainzos M, Gonzalez-Rodriguez FJ. Necrotizing soft tissue infections. Curr Opin Crit Care. 2007; 13(4): 433-9.

[5] Changchien CH, Chen YY, Chen SW, Chen WL, Tsay JG, Chu C. Retrospective study of necrotizing fasciitis and characterization of its associated Methicillin- resistant Staphylococcus aureus in Taiwan. BMC Infect Dis. 2011; 11: 297.

[6] Hefny AF, Eid HO, Al-Hussona M, Idris KM, Abu-Zidan FM. Necrotizing fasciitis: a challenging diagnosis. Eur J Emerg Med. 2007; 14(1): 50-2.

[7] Levine EG, Manders SM. Life-threatening necrotizing fasciitis. Clin Dermatol. 2005; 23(2): 144-7.

[8] Taviloglu K, Yanar H. Necrotizing fasciitis: strategies for diagnosis and management. World J Emerg Surg. 2007; 2: 19.

[9] Majeski J, Majeski E. Necrotizing fasciitis: improved survival with early recognition by tissue biopsy and aggressive surgical treatment. South Med J. 1997; 90(11): 1065-8.

[10] Martinelli G, Alessandrino EP, Bernasconi P, Caldera D, Colombo A, Malcovati L, Gaviglio MR, Vignoli GP, Borroni G, Bernasconi C. Fournier's gangrene: a clinical presentation of necrotizing fasciitis after bone marrow transplantation. Bone Marrow Transplant. 1998; 22(10): 1023-6.

[11] Al Shukry S, Ommen J. Necrotizing Fasciitis - report of ten cases and review of recent literature. J Med Life. 2013; 6(2): 189-94.

[12] Bhandari M, Acharekar KL, Kalla VK. Urinary extravasation and gangrene of genitalia. Indian J Surg. 1983; 45: 129-34. 
[13] Freischlag JA, Ajalat G, Busuttil RW. Treatment of necrotizing soft tissue infections. The need for a new approach. Am J Surg. 1985; 149(6):751-755.

[14] Puvanendran R, Huey JC, Pasupathy S. Necrotizing fasciitis. Can Fam Physician. 2009; 55(10) 981-7.
[15] Bilton BD, Zibari GB. Aggressive surgical management of Necrotizing fasciitis serves to decrease mortality; A retrospective study. Am Surg. 1998; 64(5): 397-401.

[16] Perez F, Hujer AM, Hujer KM, Decker BK, Rather PN, Bonomo RA. Global challenge of multidrug-resistant Acinetobacter baumannii. Antimicrob Agents Chemother. 2007; 51(10): 3471-84. 